Nouvelles perspectives en sciences sociales

Revue internationale de systémique complexe et d'études relationnelles

\title{
L'injonction à être soi-même : entre quête de singularité et standardisation
}

\section{Christian Le Bart}

Volume 8, numéro 1, novembre 2012

URI : https://id.erudit.org/iderudit/1013918ar

DOI : https://doi.org/10.7202/1013918ar

Aller au sommaire du numéro

\section{Éditeur(s)}

Prise de parole

\section{ISSN}

1712-8307 (imprimé)

1918-7475 (numérique)

Découvrir la revue

\section{Citer cet article}

Le Bart, C. (2012). L'injonction à être soi-même : entre quête de singularité et standardisation. Nouvelles perspectives en sciences sociales, 8(1), 61-81.

https://doi.org/10.7202/1013918ar

\section{Résumé de l'article}

L'injonction à être soi-même constitue une norme essentielle au sein des sociétés contemporaines. Dans un contexte de relatif déclin des identités prescrites, chacun est incité à effectuer un travail identitaire spécifique afin de découvrir une supposée identité vraie. Celle-ci, véritable mythologie de notre temps, est pensée comme tout à la fois substantialisée, cachée, et singulière. D’où l'importance des expériences identitaires par lesquelles les individus tentent de s'en approcher. On s'intéressera ici plus particulièrement, à partir d'une enquête sur les fans des Beatles, à la musique en tant que support identitaire spécifique. 


\title{
L'injonction à être soi-même : entre quête de singularité et standardisation
}

\author{
Christian Le Bart \\ Institut d'études politiques de Rennes \\ Centre de recherches sur l'action politique en Europe \\ (CRAPE)-CNRS
}

$\mathbf{U}$

n nombre significatif de travaux sociologiques contemporains développent l'idée selon laquelle les individus seraient devenus depuis une trentaine d'années, plus réflexifs ${ }^{1}$, c'est-à-dire plus volontiers disposés à se prendre eux-mêmes comme objets de réflexion. Là où jadis la réflexivité était le privilège de quelques-uns, des stoïciens à Montaigne, de Saint-Augustin à Jean-Jacques Rousseau ${ }^{2}$, la sociologie met en évidence la banalité du questionnement sur soi. Les conditions de possibilité d'un questionnement sur soi doivent être interrogées sociologiquement : positivement, il suppose une certaine disponibilité, des ressources culturelles, une disposition à l'intériorité dont Norbert Elias nous enseigne qu'elle n'est pas universelle ${ }^{3}$. Négativement,

Zygmunt Bauman, The Individualized Society, Cambridge, Polity Press, 2001; Antony Giddens, Modernity and Self-Identity : Self and Society in the Late Modern Age, Cambridge, Polity Press, 1991.

2 Charles Taylor, Les Sources du Moi. La formation de l'identité moderne, Paris, Seuil, 1998 [1989].

3 Norbert Elias, La Société des individus, (trad.), Paris, Pocket, 1991, [1987]. L'histoire longue de la réflexivité individuelle est largement marquée par le Christianisme qui a précocement incité les individus à l'introspection, à l'examen, à la communication directe avec Dieu. La Réforme accentuera particulièrement cette incitation. 
ce questionnement est activé par le déclin des identités prescrites (celles qui à la naissance disaient clairement et définitivement à l'individu qui il était) et des identités acquises offrant aux individus une identité (professionnelle, familiale...) stabilisée. Au carrefour de ces processus, la réflexivité, très ambivalente donc, se fait de plus en plus identitaire : l'individu contemporain n'est plus seulement soucieux de savoir ce qu'il doit faire, car ceci supposerait qu'il sache qui il est et ce qu'il veut; il est de plus en plus travaillé par une interrogation plus fondamentale, de type : qui suis-je vraiment? On est passé de la réflexivité instrumentale (que faire?) à la réflexivité identitaire (qui suis-je?). Dans le premier cas, l'individu se voit attribuer un jeu fermé et sécurisant d'identités (identité de genre, identité familiale, identité professionnelle, identité de classe, identité locale ou nationale, identité religieuse...), il est pris en charge par des institutions plus ou moins étouffantes mais identitairement sécurisantes. Stabilisé par ce que Pierre Bourdieu appelait un habitus, il peut développer d'un champ à l'autre une rationalité et une réflexivité instrumentale (comment s'enrichir?; comment faire carrière?; comment être un bon militant ou un bon chrétien?...). Dans le second cas, un doute plane sur ce qu'il est, et même sur qui il est. La crise des institutions ${ }^{4}$ se marque dans le refus de se satisfaire des identités imposées, perçues comme aussi arbitraires que la violence symbolique prêtée à ces institutions. Celles-ci n'offrent plus que des rôles appropriés avec distance, entre ironie et cynisme. Si chaque individu est conscient d'occuper une position sociale, la précarité objective de celle-ci interdit la prescription identitaire : les ancrages familiaux et professionnels sont objectivement (et sont perçus comme) beaucoup plus fragiles et provisoires qu'auparavant. Néocapitalisme et recompositions familiales conjuguent leurs effets pour priver les individus de la croyance selon laquelle leur identité est tout entière dans la position qu'ils occupent. Les croyances religieuses se sont effritées et la foi n'est plus synonyme de sécurité identitaire : elle est vécue comme quête, cheminement, interrogation. S'impose désormais au plus grand nombre

François Dubet, Le Déclin de l'institution, Paris, Seuil, 2002. 
le souci de savoir qui l'on est vraiment, au-delà des places distribuées arbitrairement et provisoirement. C'est ce questionnement identitaire que nous allons étudier : il est marqué par le désir de transparence à soi-même. Les individus, pris dans le processus d'individualisation ${ }^{5}$, veulent se connaître, savoir qui ils sont vraiment, découvrir leur identité authentique. Celle-ci ne peut être que singulière: quand les identités prescrites par les institutions sont impersonnelles (statuts, rôles...), l'identité découverte " au fond de soi » est, par hypothèse, singulière. C'est du moins ce que pensent les individus. Car, en réalité, les choses sont bien sûr plus compliquées : le "moi profond " n'est évidemment pas ce trésor caché singulier qu'espéraient les individus plongés dans la réflexivité identitaire ${ }^{6}$.

Nous montrerons que le " moi profond ", l'identité vraie, constitue un mythe contemporain très prégnant ${ }^{7}$. Il nourrit par exemple les industries culturelles de masse. Il mobilise fortement les individus, soucieux bien au-delà de l'adolescence de savoir qui ils sont. Nous étudierons les stratégies développées par ces individus pour faire aboutir la recherche de soi, recherche dont nous montrerons que paradoxalement elle suppose toujours le détour par autre chose que soi : des personnes, des objets, des choses, plus généralement encore des expériences identitaires, c'est-à-dire des occasions offertes à l'individu d'éprouver ce qu'il croit être la réalité ultime de sa personne. Nous montrerons que ces expériences sont toujours partielles et inabouties, comme s'il demeurait toujours une part d'opacité à soi-même. C'est alors la vie entière, et non plus seulement le temps de l'adolescence, qui est constitutive de la recherche de soi. Nous terminerons sur l'instrumentalisation par les institutions de cette mythologie identitaire. Un temps fragilisé, l'État s'adapte à la nouvelle donne

$5 \quad$ Ulrich Beck et Elisabeth Beck-Gernsheim, Individualization, Londres, Sage, 2001; Vincent de Gaulejac, Qui est "je”?, Paris, Seuil, 2009.

6 François Flahault, Be Yourself! Au-delà de la conception occidentale de l'individu, Paris, Mille et une nuits, 2006; Jean-Claude Kaufmann, L'Invention de soi : une théorie de l'identité, Paris, Armand Colin, 2004.

7 Le terme de mythe est ici utilisé au sens de Roland Barthes (Mythologies, Paris, Seuil, 1957) : il est croyance naturalisée intériorisée comme évidence. 
identitaire. De même le capitalisme, pour qui le contexte de réflexivité identitaire constitue une double opportunité : de nouveaux marchés se créent autour de nouveaux besoins postmatérialistes; et de nouvelles formes de dominations dans le travail sont rendues possibles par la requalification de celui-ci en expérience identitaire. On verra alors s'esquisser le contre-modèle d'une subjectivité paradoxalement très standardisée.

Nous nous appuierons pour donner chair à cette argumentation sur un matériau d'entretiens collecté il y a une quinzaine d'années auprès de fans des Beatles ${ }^{8}$. Le rapport à la musique en général constitue sans doute un terrain particulièrement riche pour étudier la réflexivité identitaire, pour peu que l'on dépasse la pure consommation récréative. À partir du moment où il y a investissement passionnel, on peut s'attendre à ce que la problématique identitaire soit pertinente. Cette enquête auprès de passionnés ne suffira évidemment pas à conclure définitivement sur des questions aussi lourdes. Notre corpus d'entretiens (une trentaine de passionnés Français et Belges) est trop restreint et sans doute trop spécifique pour autoriser une montée en généralité brutale à l'échelle des sociétés contemporaines en général. Au moins permettra-t-il de donner consistance empirique à nos hypothèses. Ce qui suppose au passage (c'est un point essentiel) de considérer les fans comme des gens ordinaires, des acteurs sociaux comme les autres, bien loin donc des stéréotypes alimentés par les médias. La passion musicale (le terme de "fan " est presque toujours récusé par les enquêtés) est une passion ordinaire' la passion Beatles touche des gens appartenant à toutes les classes sociales, aux deux genres, à toutes les générations. Elle se conjugue à toutes les positions sociales, tous les systèmes de goûts ou de dispositions. À l'inverse donc des journalistes sensationnalistes qui aiment à faire de l'attitude fan une pathologie (adolescente) et du fan un quasi monstre, notre objectif est tout au

$8 \quad$ Christian Le Bart (avec Jean-Charles Ambroise), Les Fans des Beatles, Rennes, Presses universitaires de Rennes, 2000; Christian Le Bart, « Stratégies identitaires de fans : l'optimum de différenciation ", Revue française de sociologie, vol. 45, n 2, 2004, p. 283-306.

9 Christian Bromberger, Passions ordinaires, Paris, Bayard, 1998. 
contraire d'observer chez les passionnés des mécanismes de construction identitaire typiques des sociétés contemporaines. Sans doute la passion, en ses formes exacerbées, donne-t-elle à ces mécanismes un relief spécifique qui en facilite l'observation : mais celui-ci ne mérite examen que s'il vaut au-delà des personnes rencontrées, c'est en tous cas le sens de notre travail.

\section{Les expériences identitaires comme découvertes de soi}

La réflexivité identitaire se marque dans le souci de se connaître, d'aborder la vie en sachant qui on est vraiment, comme s'il s'agissait de dépasser un rapport à soi originellement frappé du principe d'opacité. Les expériences de plaisir et de déplaisir sont aujourd'hui lues comme autant de pistes et d'indicateurs permettant à l'individu de savoir s'il est sur la bonne piste, celle qui conduit à sa nature vraie et à son identité enfouie. L'épanouissement dans telle activité, le sentiment de plénitude et d'authenticité, l'impression d'être soi, tout cela donne lieu à analyses réflexives.

Il nous semble donc possible de lire comme expériences identitaires des comportements qui ont comme caractéristiques d'être valorisés à partir du moment où ils donnent aux individus le sentiment de solliciter leur personnalité vraie et singulière. Là où, par exemple, le simple goût pour les Beatles distrait, change les idées, procure une sensation agréable mais éphémère ("j’aime bien »), la véritable passion permet à l'individu d'accéder à la vérité ultime de sa personne. L'écoute devient expérience identitaire quand elle est ritualisée, sacralisée, mise en scène, singularisée. Elle gagne en densité :

Je peux pas écouter les Beatles en faisant autre chose. Si je fais du ménage et que j'écoute les Beatles, c'est réducteur pour eux. Si je bosse en écoutant les Beatles, je peux pas bosser. Faut voir que moi, depuis la quatrième, chaque morceau des Beatles, il y en a entre 200 et 250, je les ai décortiqués note par note, instrument par instrument, avec des bouquins et tout ça. Non, je peux pas les réduire à ça (Sébastien, 22 ans, étudiant en histoire).

Ainsi des fans racontant les petites cérémonies du plaisir qui organisent l'écoute : enfermement dans un espace clos, souvent sans lumière, écoute au casque... plus rien ne doit exister entre 
soi et la musique. La communion doit être directe entre le fan et la musique, alors seulement la plongée en soi-même est possible.

J'aimais bien me mettre dans le noir, je fermais la porte, je mettais la musique, je me couchais sur le divan, totalement dans la musique pour pouvoir m'imprégner... Et au bout d'un moment, j'avais plus besoin de les écouter, je les avais emmagasinés (Yann, 17 ans, lycéen).

L'individu n'est plus un personnage social, il devient individu pur. Il n'a plus d'âge (retour à l'adolescence pour les plus âgés), il n'a plus de rôle social (tous les attributs identitaires sont neutralisés). Face à lui, la musique devient pure musique : elle n'est plus située dans le temps, elle n'a plus de signification. La communion et le sentiment d'enfin être soi surgissent de l'apesanteur sociale.

La musique se voit conférer un pouvoir qui s'apparente au pouvoir de la magie. Au-delà des discours convenus sur l'émotion musicale, sur les souvenirs qui lui sont associés, sur la beauté intrinsèque des mélodies, sur le pouvoir évocateur d'un langage sans signifié, tous les passionnés reconnaissent leur impuissance à décrire précisément ce qui se passe en eux lorsqu'ils opèrent cette plongée en soi-même via la musique. La révocation du langage pour décrire une expérience perçue comme indicible (d'où les longs silences pendant les entretiens) signe plus généralement la défaite de tous les artifices sociaux lorsqu'est en jeu l'identité vraie. Parce que celle-ci n'est pas sociale, les outils sociaux comme le langage sont impuissants à en rendre compte. L'identité vraie peut être approchée, entraperçue, mais toujours elle se dérobe :

J'ai souvenir d'avoir lu les paroles d'une chanson et d'avoir ressenti... que ça traduisait quelque chose. Quelque chose de vachement bien. Que je ressentais. Et puis je l'ai relue une deuxième fois et là ça ne m'a plus rien fait. Je trouvais ça banal, tout de suite après. C'est bizarre. Se dire que ces gens, ils ont les mêmes sentiments que toi (Yann, 17 ans, lycéen).

Il serait réducteur de croire que la simple écoute suffit à réaliser immédiatement et mécaniquement cette rencontre avec soimême. L'expérience ne peut être qu'exceptionnelle tant elle suppose de conditions permettant la mise entre parenthèses 
efficace du social. Celui-ci peut resurgir et brouiller l'expérience identitaire : ainsi les chansons trop connues des Beatles, tombées dans le domaine public, comme souillées par les usages collectifs, sont délaissées des fans qui leur préfèrent des morceaux rares, moins exposés, moins diffusés. La quête du sens caché autorise alors les expériences les plus originales visant à rompre avec l'écoute classique :

Tu prends Helter Skelter. Un pote m'avait dit : tu tournes à l'envers ${ }^{10}$, tu entends : turn me on dead man (excite-moi, homme mort). J'avais essayé de le faire. Dead man étant soit disant, dans l'ésotérisme satanique, le Christ (Joël, 29 ans, enseignant d'histoire-géo).

Se profile l'idéal du morceau qui n’aurait été composé que pour soi, qui n'aurait pas de réalité au-delà de l'appropriation par le fan et lui seul. Au fond, la plongée en soi-même ne peut être qu'exceptionnelle car elle repose en dernière analyse sur l'illusion d'une musique créée, interprétée, diffusée, pour soi seul. Le mythe de l'identité singulière suppose en effet que le fan soit interpellé comme être singulier par un morceau lui-même singulier. Plusieurs fans aiment à croire, contre la plus élémentaire vraisemblance sociologique, que tel morceau n'existe que pour eux, qu'eux seuls le connaissent, qu'il correspond si intimement à ce qu'ils sont vraiment qu'il a comme été écrit pour eux. Bien sûr, tous savent que les morceaux religieusement et solitairement écoutés sont en réalité diffusés à des millions d'exemplaires par une industrie culturelle planétaire. Tous font l'expérience (toujours douloureuse) de la rencontre avec d'autres fans étrangement semblables dans l'expression de leur passion. Mais tous aiment à recréer les illusions d'une relation intime et personnelle entre le musicien et eux. Dans l'espace enchanté et clos de la chambre d'adolescent, tout devient possible : "J'avais l'impression d'être leur copain ", se souvient par exemple Éric (38 ans, policier).

Tout se passe au fond comme si la découverte de la singularité en soi ne pouvait se faire que par l'intermédiaire de musiques elles-mêmes singulières. Et les fans ne manquent pas de ressources

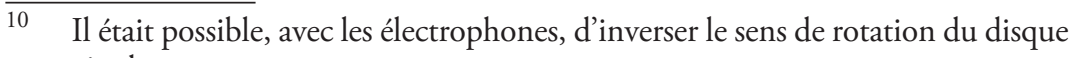
vinyle. 
pour transformer les produits manufacturés les plus formatés en objets individuels singuliers : ainsi la tactique consistant à fabriquer pour soi l'album idéal des Beatles, en un seul exemplaire évidemment, à partir des chansons que l'on préfere, et si possible dans l'ordre de préférence. On a là un bel exemple de bricolage permettant le passage du standardisé au singulier.

L'exemple de la passion musicale est intéressant car il permet de mettre en évidence une donnée centrale pour qui veut saisir les expériences identitaires. Si celles-ci comportent forcément une dimension de réflexivité individuelle (qui suis-je?), cette réflexivité ne tourne jamais à vide comme pur exercice de réflexion sur soi. L'angoisse identitaire, dont nous avons dit qu'elle était nourrie par le déclin des grands ancrages sociétaux, ne trouve pas sa solution dans une réflexivité close et solitaire. Elle se nourrit de comportements qui toujours supposent le détour par des supports. C'est la musique qui joue ici ce rôle : elle fonctionne comme un miroir qui permet à l'individu de savoir qui il est. Seul, celui-ci n'y parviendrait pas par le simple jeu de sa réflexivité.

Le terme support n'a ici d'autre intérêt que de permettre de ranger sous une même rubrique des éléments en réalité très différents, aussi différentes que les expériences identitaires ellesmêmes. Quittons un instant le terrain musical : la sociologie étudie par exemple la façon dont le couple est aujourd'hui principalement défini comme lieu d'affirmation de soi non pas contre l'autre ou indépendamment de l'autre, mais bien grâce à lui. L'idéal de réflexivité n'est pas fourni par le célibat mais bien par la relation amoureuse. Comme l'a montré François de Singly ${ }^{11}$, c'est au contact de l'être aimé que je me sens moi-même, que je sais qui je suis. L'être aimé est le plus court chemin qui mène à soi.

Si donc il ne saurait y avoir d'expérience identitaire sans réflexivité, celle-ci ne saurait se concevoir comme une pure

11 François de Singly, Le Soi, le couple, la famille, Paris, Nathan, 1996; L'Individualisme est un humanisme, La Tour d'Aigues, Éditions de l'Aube, 2005. 
relation de l'individu avec lui-même. C'est même un paradoxe de la seconde modernité que de toujours exiger ces supports que sont les autres individus (autrui significatif), les objets, les actions. S'agissant de ces dernières, il faut constater à quel point aujourd'hui les registres d'action privilégiés par les individus sont ceux qui peuvent se conjuguer au désir de s'épanouir, de s'exprimer, d'être soi-même. C'est vrai y compris au niveau professionnel : la rémunération financière ne saurait étouffer l'attente d'une rétribution identitaire. Contre le travail aliénant qui détourne de soi, les salariés attendent aujourd'hui du travail qu'il leur corresponde vraiment. On retrouve a fortiori ce type d'exigence au niveau des loisirs. Le succès des pratiques créatives (arts plastiques, écriture, musique...) s'accompagne d'un besoin décomplexé de s'exprimer, indépendamment de toute prétention à faire œuvre d'art. On peint ou on écrit pour soi, sans prétendre à un talent particulier, sans vouloir en imposer aux autres, juste pour être soi. Les sensations fournies par le sport s'analysent dans les mêmes termes : la performance compte moins que le face-à-face avec soi-même. Il s'agit de se faire plaisir. Le goût du risque, le désir d'aller " au bout de soi-même ", d'éprouver ses limites, tout cela participe du mythe de l'expérience vraie et authentique. Les pratiques historiquement construites autour de l'idée de renoncement à soi ou d'abandon de soi sont ainsi redéfinies. C'est le cas du militantisme, désormais traduit dans le langage de l'accomplissement de soi et de l'épanouissement personnel. Il ne s'agit plus de faire le sacrifice de soi au profit d'une cause qui dépasse (et délégitime) les préoccupations individuelles; il s'agit de donner de soi (de son temps, de son argent...) pour au final être soi, être en accord avec soi-même. Même chose sur le plan de l'engagement religieux.

Si cette quête de soi se déploie en une infinité de pratiques, si elle s'adosse à une pluralité de supports, c'est bien sûr parce qu'elle est marquée du principe fondamental d'inachèvement. Elle s'inscrira donc dans la longue durée d'une trajectoire biographique irréductible aux incertitudes de l'adolescence. Les fans de Beatles en témoignent à tous les âges. La quête est sans fin, à 
mesure que l'industrie culturelle propose de nouveaux enregistrements, sur de nouveaux supports :

Je suis quelqu'un qui aime bien répartir son plaisir. Donc j'essaie de me discipliner pour ne pas tout acheter en une fois [...]. Donc j'ai étalé. J'ai découvert petit à petit chaque album (Yvon, 34 ans, psychologue).

Cette dimension mobilisatrice à long terme autorise à parler de mythe. Non pas seulement parce que l'identité vraie, substance cachée, est une illusion sans fondement d'un point de vue sociologique; mais aussi parce que la croyance en cette substance mobilise les individus tout au long de leur vie. L'intranquillité identitaire, longtemps cantonnée à un âge de la vie (l'adolescence) ou à certains secteurs de la vie sociale (la bohème et les mondes de l'art) tend aujourd'hui à s'universaliser. L'individu stylisé décrit par Pierre Bourdieu dans La Distinction, largement stabilisé autour d'un habitus de classe dominant (voire hégémonique), tend à devenir l'exception. C'est au contraire l'habitus clivé qui devient le modèle dominant. L'inquiétude propre aux individus pluriels $^{12}$, pris entre des socialisations multiples, dépositaires d'habitus incompatibles, se retrouve dans tous les univers sociaux et ne disparaît plus du fait d'une désormais improbable installation. Parce qu'ils sont objectivement et subjectivement provisoires, les ancrages familiaux et professionnels ne suspendent que très partiellement l'intranquillité identitaire. La quête de soi, enrichie sans cesse de nouvelles expériences, n'a pas de fin. Les individus ont l'impression de ne jamais parvenir complètement à savoir qui ils sont vraiment, comme si l'identité vraie, approchée, se dérobait toujours. En termes sociologiques, il est facile de constater que la recherche elle-même rebat les cartes, déplace objectivement les identités individuelles, celles-ci avançant au même rythme que ceux qui les poursuivent. Toute expérience identitaire fabrique de nouvelles facettes identitaires dont la synthèse est à refaire en un cycle infini d'expériences (qui apportent de nouvelles dimensions) et de réflexivité (qui fait la synthèse des expériences vécues).

$\overline{12}$ Bernard Lahire, La Culture des individus, Paris, La Découverte, 2004. 


\section{L'identité singulière comme mythe contemporain}

La croyance en l'existence d'un moi profond authentique est nourrie d'une critique ordinaire des identités prescrites imposées par les institutions. Le mouvement de mai 68 est ici une référence commode pour décrire l'accélération du processus d'individualisation opérée avec ce que certains auteurs appellent la seconde modernité. Les individus n'acceptent plus de se définir en référence aux identités que les institutions prescrivent et en référence aux seuls collectifs auxquels ces institutions les rattachent. Les institutions sont perçues comme aliénantes. Elles mutilent l'individu par une injonction violente et unilatérale à occuper la position qu'elles lui ont donnée. La thématique de l'émancipation individuelle se nourrit d'une humeur anti-institutionnelle qui récuse les réponses simplistes à la question du « qui suis-je? ». Les institutions prétendaient répondre à cette question en se contentant de dire aux individus non pas qui ils étaient, mais ce qu'ils étaient, et ce qu'ils devaient se contenter d'être (des étudiants, des Français, des hommes ou des femmes...). La rhétorique d'émancipation opère un basculement qui est évidemment, à terme, très inconfortable pour l'individu. Celui-ci révoque les institutions et doit affronter seul une question redoutable, celle de savoir qui il est vraiment. Or cette identité authentique se caractérise par trois dimensions fondamentales, qui la différencient de l'identité sociale : elle est substantialisée, quand la précédente se trouvait partiellement construite par les hasards de la distribution des positions sociales; elle est singulière, quand la précédente rattachait à des collectifs (classe, genre...); elle est enfouie, quand la précédente résultait mécaniquement des positions occupées (" je suis ouvrier ", " je suis mère de famille "). On glisse vers l'inconfort identitaire : la transparence à soi-même est une quête sans fin, il restera toujours une part d'opacité, voire de mystère.

Lidentité vraie est substantialisée, elle n'est pas à construire. Chaque individu, selon les représentations aujourd'hui dominantes, est doté d'une nature, celle-ci l'habite de sa naissance à sa mort, elle est une substance inchangée. Les aléas biographiques 
ne peuvent altérer cette substance, l'individu restera toujours lui-même. Il change superficiellement, sa nature profonde constitue la part de lui-même sur laquelle les événements n'ont pas prise. On se gardera bien d'interroger plus avant cette nature première de l'individu : patrimoine génétique? Configuration psychologique liée aux expériences définitives de la petite enfance? L'approximation n'entame pas le mythe de l'identité vraie. Il s'inscrit en tous cas clairement contre la vision constructiviste (sociologique) de l'identité. Au fil des expériences identitaires dont il pense qu'elles le mettent au contact de lui-même, l'individu n'éprouve pas le sentiment de se construire, mais de se découvrir. L'identité est déjà là, comme un trésor enfoui, qu'il s'agit d'aller chercher. Au passage, on notera l'incompatibilité entre la vision ordinaire et mythique de l'identité et la vision sociologique. Les sciences sociales sont porteuses sur ces questions d'une vision désenchantée : l'individu n'a d'autre substance que ce que la société a déposé en lui, le trésor caché est vide, la quête identitaire n'a d'autre finalité qu'elle-même, et c'est au fil de celle-ci que s'élabore ce qui en fera l'objet, le sentiment d'être soi.

Les entretiens permettent de saisir cette dimension première de l'identité. Ainsi lorsque la musique se voit créditer du pouvoir de solliciter en soi quelque chose d'enfoui, une sorte de déjà-là insoupçonné :

J'ai une image très précise en tête. J'avais 14 ans. Et un jour, j'ai vu des extraits musicaux de cette époque. Qui rejoignaient quelque chose. Il y avait les Beatles, en noir et blanc. Et là je me suis dit : tiens, j'aime déjà ça. Donc ça veut dire que c'est antécédent. Quelque chose de déjà là (Bernard, 35 ans, régisseur d'un centre culturel).

Au-delà de la musique, les personnages Beatles, en tant que figures exemplaires et idéales-typiques offrent les clés d'une réflexivité identitaire particulièrement féconde. On voit se déployer des formes subtiles d'identifications partielles :

Au lycée, on était quatre. J'avais l'impression d'être le McCartney du groupe. Autour du sport. Mais j'ai aussi l'impression que, dans mon comportement, j'ai un peu des quatre. Le fait de s'imposer, d'être chef du groupe, c'est mon tempérament, c'est McCartney. Le côté rêveur de G. Harrison, le caractère plus violent et impulsif de Lennon, et la bonne 
humeur de Ringo. Je pense que c'est un peu ça (Nicolas, 21 ans, étudiant).

Avec Sébastien, lui c'était Lennon, il avait la voix de Lennon, tout le monde le disait. Donc, moi, j'étais McCartney. En gros, c'est con à dire, mais c'est ça (Thierry, 21 ans, étudiant).

L'identité vraie est ensuite supposée cachée, enfouie sous les masques multiples des socialisations successives. Ces socialisations, institutionnelles (école, famille, travail...) assignent aux individus des rôles fondamentalement inauthentiques. La relation entre l'individu et la société, perçue comme un rapport de force frontal, est forcément défavorable au premier. Celui-ci doit, pour accéder à la part de singularité qui est en lui, se débarrasser de ce qui n'est pas lui. Il doit se défaire de tout ce que la société a déposé en lui, pour espérer entrevoir ce qu'il est vraiment, c'està-dire ce qu'il était en amont de toute socialisation, ce qu'il ne cessera jamais d'être mais qu'il peut ignorer s'il se contente des rôles et des places que la société lui a assignés. Dans ce contexte, l'enfance est évidemment valorisée : elle est le moment de vérité de la personne, en amont de sa prise en charge par la société (inné versus acquis). Sont également valorisés les moments vrais liés aux émotions fortes et premières (ici la détresse liée à la maladie d'un proche), émotions auxquelles la musique des Beatles donne un écho particulier :

J'ai surtout apprécié les Beatles à partir du moment où mon mari est tombé malade. Quand j'écoutais, ça me faisait quelque part une douleur, des sensations un peu douloureuses mais que j'appréciais quand même parce que ça me permettait d'extérioriser cette douleur que j'avais en moi. La musique m’emportait et me créait certaines sensations [...]. Beaucoup des musiques des Beatles traduisent la mélancolie, la tristesse. Et ça me frappait parce que j'étais dans cet état-là aussi (Christine, 58 ans, femme au foyer).

Le décalage entre le soi authentique, unique, et les rôles sociaux, multiples et changeants, se mesure, toujours si l'on suit les individus, à la souffrance qu'ils ressentent dans l'accomplissement de ces rôles. Cette souffrance est proportionnelle à la distance qui sépare la vraie nature supposée de la personne et le rôle qu'on lui fait jouer. De ce fait, la souffrance sert aussi désormais 
d'indicateur d'authenticité : si je suis bien avec tel nouveau compagnon, si je me sens bien dans tel nouveau travail, si les cours de telle filière me plaisent, c'est qu'ils me correspondent, qu'ils me mettent au contact de moi-même. Par opposition à cela, la pénibilité des rôles qui obligent l'individu à se faire violence révèle un écart insurmontable. Dès lors, il importe que l'individu soit à l'écoute de lui-même : si quelque chose résiste en lui, c'est qu'il est dans un rapport d'inauthenticité qui, à terme, est sans solution. Là où, jadis, les individus anticipaient sur une possible socialisation (" tu t'y feras »), ils considèrent désormais que le moi vrai n'est pas déplaçable à l'infini. On ne lui fait pas impunément violence.

L'identité vraie est enfin singulière. La seconde modernité universalise l'injonction romantique à se libérer d'une rationalité standardisée car universelle, celle des Lumières. Réhabilitant la pluralité des passions au détriment de l'unidimensionnalité de la Raison, les romantiques se rebellent contre l'ordre bourgeois qui réduit l'individu. Systématisée au sein du monde de l'art, la disposition à travailler sa singularité devient un mot d'ordre recevable dans l'ensemble de la société. L'opposition ancienne entre l'univers de la bohème, où chacun est libre d'être lui-même, et l'univers bourgeois, où chacun se doit d'être conforme aux rôles qui lui sont assignés, est aujourd'hui dépassée. Comme l'ont montré aussi bien les travaux de Nathalie Heinich ${ }^{13}$ que ceux de Luc Boltanski et Eve Chiapello ${ }^{14}$, les règles du jeu spécifiques au monde de l'art, en particulier l'injonction à être soi sous une forme singulière, ont progressivement colonisé les autres champs sociaux. L'élève ou le salarié sont incités à mettre d'eux-mêmes dans leur travail. Leur personnalité vraie, qui déborde nécessairement les rôles standardisés qu'ils endossent, doit pouvoir s'exprimer au sein de l'institution. Chaque élève est différent, a son propre rythme, sa personnalité, il est enfant avant d'être élève. Il n'est plus cette mire molle à partir de laquelle l'école standardisée

13 Nathalie Heinich, L'Élite artiste, Paris, Gallimard, 2005.

14 Luc Boltanski et Eve Chiapello, Le Nouvel esprit du capitalisme, Paris, Gallimard, 1999. 
fabriquera en série des élèves citoyens aussi semblables que possible, aussi conformes que possible à l'idéal républicain; il est un enfant singulier, différent des autres, vis-à-vis de qui l'école devra se mettre à l'écoute pour nouer un dialogue. Il y a dialogue ou rapport de force là où auparavant prévalaient le monologue et l'injonction unilatérale. L'enfant se voit doté d'une singularité originelle : ce n'est pas l'ordonnancement spécifique des socialisations au carrefour desquelles il est placé qui le singularise, c’est sa nature première d'être singulier. Il l'est en amont de toute socialisation. Là où la sociologie assimile la singularité des individus à la rareté statistique des expériences vécues, le mythe contemporain de l'individu demeure relativement indifférent à ces expériences : elles peuvent jouer sur la capacité de l'individu à savoir qui il est vraiment; elles ne peuvent pas jouer sur qui il est vraiment.

Les entretiens avec les fans des Beatles constituent un excellent matériau pour observer cette quête de singularité. Ceux-ci sont en effet confrontés à une difficulté spécifique. Comme le suggère le terme de fan, le risque existe pour chacun d'entre eux de n'être qu'un fan parmi d'autres, semblable aux autres dans la prétention dérisoire à imiter les Beatles. L'imitation pure est simple (s'habiller comme..., se coiffer comme... ${ }^{15}$ ) est récusée, souvent avec énergie, comme aliénation. Mais le simple fait que les fans se comptent par milliers n'interdit-il pas toute prétention à la singularité? Certains s'efforcent de purement et simplement ignorer leurs semblables : "J'avais découvert les Beatles, et ça n’était qu’à moi » raconte ainsi Bernard (35 ans, régisseur d'un centre culturel). La présence des autres finit pourtant toujours par s'imposer, par exemple à l'occasion des concerts, expérience souvent vécue comme ambivalente.

Les concerts, il y a trop de monde. Moi, j'aimerais bien être seule à la limite. Toi, tu y vas de façon religieuse, et puis il y a des petits cons qui chantent; ça, ça m'énervait (Fabienne, 39 ans, enseignante d'anglais).

Le concert, c'était merveilleux, mais en même temps j'étais déçue [...].

$\overline{15}$ Le "fan de Johnny " ou " le fan de Claude François " sont invariablement convoqués comme figures repoussoir. 
Je me suis retrouvée avec des milliers de gens, loin de la scène [...]. J'ai pas voulu partager cette passion avec d'autres. J'étais déçue de me retrouver si petite parmi tant de personnes. Je le croyais mon confident. Et puis là, bon, il était à tout le monde (Alice, 30 ans, enseignante d'anglais).

Cette déception nourrit des stratégies de repli sur l'intime. Chacun reconstruit ses Beatles à soi, et c'est sur cette base que l'identité singulière peut refaire surface. À chacun de se singulariser dans l'univers Beatles, par la possession d'objets de collections, par des publications ou des compositions personnelles, par l'expression de préférences paradoxales, par une érudition distinctive, ou tout simplement par l'enfermement dans l'intimité de la musique. Les Beatles sont universels, mais chacun fabriquera ses Beatles à soi.

\section{Singularité ou standardisation?}

Le modèle de l'individu stratège poursuivant une quête identitaire sans fin présente des vertus descriptives et même compréhensives. Travaillé par le mythe de l'identité vraie, l'individu développe une réflexivité stratégique qu'il est sociologiquement possible de suivre à la trace, en particulier à partir d'entretiens. Ces derniers constituent en effet en eux-mêmes des moments forts de réflexivité identitaire. Pour autant, la recherche risque de s'enfermer dans une impasse si elle reste à hauteur d'individu et s'interdit de voir au-delà des stratégies individuelles. L'explication doit enrichir la compréhension et mettre en lumière les conditions sociales de production de ces stratégies. Contre l'illusion d'une réflexivité qui serait face-à-face avec soi-même, il faut rappeler non seulement le rôle des supports qui rendent possible ce face-à-face, et qui débordent nécessairement l'individu, mais également celui des institutions tirant parti de cette réflexivité généralisée. Deux institutions retiendront notre attention, bien au-delà donc de la sphère musicale, le néocapitalisme et l'État. L'un et l'autre fonctionnent comme de puissants dispositifs d'incitation à la réflexivité identitaire. L'État et le capitalisme se sont historiquement construits sur un affaiblissement des sociétés 
communautaires, affaiblissement dont ils furent tout à la fois la cause, le symptôme et l'effet. Mais l'un et l'autre ont dans un premier temps, celui de la première modernité, fabriqué un individu plus générique que différencié. Côté État, ce sont les figures du citoyen, de l'élève, du fonctionnaire, de l'électeur, du contribuable...; côté capitalisme, du marchand, de l'actionnaire, de l'entrepreneur, du salarié, du client... Individuation plus qu'individualisation, disons-nous ailleurs ${ }^{16}$. $\grave{A}$ chaque fois, la division du travail s'effectue jusqu'à faire exister des individus, mais jamais ceux-ci ne sont incités à faire preuve de réflexivité identitaire ou de singularité différenciée. Ils sont pris en charge par les institutions qui les distribuent en champs, en rôles, en identités.

L'État contemporain et le néo-capitalisme interpellent les individus d'une façon différente ${ }^{17}$. Ils doivent faire avec (en même temps qu'ils en précipitent l'avènement) des individus engagés dans un jeu de quête identitaire, comme on l'a dit. Le désir d'être soi, à l'écart des rôles et contre les institutions, est potentiellement déstabilisateur pour ces dernières. Elles ne peuvent dès lors se reproduire qu'à condition de jouer le jeu de la seconde modernité. L'État doit concéder à l'individu le droit d'être singulier, le néocapitalisme va plus loin : il l'instrumentalise.

L'État (par exemple providence) s'épuise à construire des catégories que les individus perçoivent désormais presque plus comme aliénantes que comme protectrices. D'où la mutation du discours des institutions étatiques, qui ne peuvent maintenir leur position de force qu'à condition de concéder à l'individu le droit d'être lui-même. L'élève s'efface au profit de l'enfant, auquel l'institution scolaire reconnaît le droit de disposer de son propre rythme biologique, de sa propre personnalité, de son propre système de goût et de préférences, etc. Il va sans dire que cette

\footnotetext{
16 Christian Le Bart, L'Individualisation, Paris, Presses de Sciences Po, 2008.

17 Etat et néocapitalisme ne sont évidemment pas des " acteurs " agissants. Ils n'existent, contrairement à ce que nos formulations trop hâtives pourraient laisser supposer, que par l'intermédiaire d'institutions et de personnes qui, elles, sont réelles : entreprises et entrepreneurs, décideurs politiques et fonctionnaires, etc.
} 
recomposition est coûteuse pour l'institution : les enseignants n'ont plus face à eux une classe d'élèves mais un groupe d'enfants, tous singuliers, tous autorisés à se faire entendre comme singuliers. Le même raisonnement vaut pour toutes les institutions d'État. Les politiques publiques se déclinent désormais sur le mode d'une proximité dont le face-à-face avec l'individu singulier est l'horizon définitif. La rhétorique du cas par cas imprègne l'action publique, comme si chaque individu cible de celle-ci était en droit d'en négocier le contenu en fonction non plus seulement de ce qu'il est mais de qui il est. Une telle recomposition du rôle de l'État modifie en profondeur la relation bureaucratique. Mais le cas par cas est aussi pour l'État prétexte à systématiser des procédures de surveillance individualisées. Au final, le diagnostic d'affaiblissement des institutions n'est donc pas évident. En se posant en partenaires et en dispositifs incitant à la réflexivité identitaire, elles imposent la transparence (à soi-même et aux autres) comme norme. Au point de sanctionner les attitudes de réserve ou de quant-à-soi autrefois valorisées mais désormais décalées. L'école sanctionnait hier l'enfant refusant de faire l'élève, il n'est pas absurde de faire l'hypothèse qu'aujourd'hui elle sanctionnerait plutôt l'élève refusant de faire l'enfant, c'est-à-dire refusant de s'engager dans des activités " créatives ", de s'exprimer "librement ", de formuler un projet d'orientation en phase avec sa "personnalité ", etc.

Le capitalisme a opéré une conversion de même ordre. Aux salariés et consommateurs conformes et dociles, clairement assignés à des positions et des rôles, il préfère désormais des individus détachés, mobiles, identitairement flottants, soucieux de singularité plus que de conformisme. Les entreprises jouent aujourd'hui la carte de l'épanouissement par le travail. Ceci suppose qu'elles offrent à ces individus des marges de manœuvre nouvelles, en pariant sur l'initiative, l'innovation, la créativité. Mais en retour, elles tirent profit d'un engagement plus total des personnes. À même de s'épanouir au travail, de s'y éclater, d'y être pleinement lui-même, le salarié engagera sa personne tout entière et non plus seulement sa force de travail. Le surtravail 
(disponibilité totale des cadres supérieurs par exemple) est rétribué en gratifications identitaires. Le travail est censé conduire à soi, au plus loin donc de l'accusation d'aliénation d'antan. Le salarié peut afficher sa singularité, il est partiellement libéré du " carcan » des statuts ou des organisations ${ }^{18}$.

Même illusion, mêmes idéologies au niveau des consommateurs. Loin des critiques de la société de consommation, qui montraient que celle-ci était un moule qui formatait les individus, le marketing a beau jeu aujourd'hui de rappeler que l'acte de consommation est multiple, libre, ouvert. Il ne fabrique plus des individus en série, il offre à chacun la possibilité de se trouver par l'intermédiaire d'un marché inépuisable de biens et de services. Le néocapitalisme offre une infinité de supports identitaires : des choses (vêtements, bijoux, déco, meubles et immeubles, livres, CD...), des voyages, des activités diverses... À chacun de se singulariser à partir des produits manufacturés. Si ma maison est remplie d'objets tous fabriqués en série, elle ne ressemble malgré tout à aucune autre par l'agencement spécifique que j'y établis. La consommation, lorsqu' elle ne sert plus à répondre aux besoins fondamentaux (se loger, se nourrir, se vêtir...), obéit à une fonction identitaire. Elle est expérience identitaire, elle permet à chacun de s'éprouver, de s'expérimenter sous une pluralité de styles. La même logique explique le succès des médias centrés sur le travail identitaire : livres dits de " développement personnel ", magazines axés sur la psychologie, le bien-être, l'épanouissement de la personne, émissions de télévision centrées sur l'intime... À chaque fois, il s'agit d'aider les individus à effectuer un travail identitaire sur eux-mêmes en leur fournissant des modèles, des exemples, en mettant à leur portée le vocabulaire semi-savant d'une psychologie ou d'une psychanalyse appropriables par tous.

Là où jadis la société fournissait l'identité par une socialisation le plus souvent unilatérale, les individus se voient à présent placés dans une situation paradoxale : la société leur impose d'être eux-mêmes, de savoir jouer le jeu de l'authenticité et de la personnalité vraie, elle est traversée par d'incessantes injonctions à

$\overline{18}$ Vincent de Gaulejac, La Société malade de la gestion, Paris, Seuil, 2005. 
être soi; et en même temps elle abandonne largement ces mêmes individus à un bricolage identitaire qu'ils doivent accomplir eux-mêmes. L'identité était donnée telle quelle, prête à être endossée; elle est désormais à construire, la société ne fournissant plus que les pièces d'un puzzle à jamais inachevé. La singularité est devenue une norme sociale forte : les déviants, ceux qui restent enfermés dans les rôles d'antan (ceux qui s'y croient, ceux qui manquent de recul et d'humour, ceux qui demeurent premier degré) ont toutes les chances de rester en marge, de même ceux qui ne parviennent pas à faire la synthèse de leurs expériences identitaires (instables, pas fiables, difficiles à cerner...). Même si, au final, la singularité est un idéal (?) inaccessible, la société exige de nous que nous sachions prendre au sérieux cet idéal et donner les signes de sa réalisation.

\section{Bibliographie}

Barthes, Roland, Mythologies, Paris, Seuil, 1957.

Bauman, Zygmunt, The Individualized Society, Cambridge, Polity Press, 2001.

Beck, Ulrich et Elisabeth Beck-Gernsheim, Individualization, Londres, Sage, 2001.

Boltanski, Luc et Eve Chiapello, Le Nouvel esprit du capitalisme, Paris, Gallimard, 1999.

Bromberger, Christian, Passions ordinaires, Paris, Bayard, 1998.

Dubet, François, Le Déclin de l'institution, Paris, Seuil, 2002.

Elias, Norbert, La Société des individus, (trad.), Paris, Pocket, 1991 [1987].

Flahault, François, Be Yourself? Au-delà de la conception occidentale de l'individu, Paris, Mille et une nuits, 2006.

Gaulejac, Vincent de, La Société malade de la gestion, Paris, Seuil, 2005.

Gaulejac, Vincent de, Qui est “je”?, Paris, Seuil, 2009.

Giddens, Antony, Modernity and Self-Identity: Self and Society in the Late Modern Age, Cambridge, Polity Press, 1991.

Heinich, Nathalie, L'Élite artiste, Paris, Gallimard, 2005. 
Kaufmann, Jean-Claude, L'Invention de soi : une théorie de lidentité, Paris, Armand Colin, 2004.

Lahire, Bernard, La Culture des individus, Paris, La Découverte, 2004.

Le Bart, Christian (avec Jean-Charles Ambroise), Les Fans des Beatles, Rennes, Presses universitaires de Rennes, 2000.

Le Bart, Christian, "Stratégies identitaires de fans : l'optimum de différenciation ", Paris, Revue française de sociologie, vol. 45, n 2, 2004, p. 283-306.

Le Bart, Christian, L'Individualisation, Paris, Presses de Sciences Po, 2008. Singly, François de, Le Soi, le couple, la famille, Paris, Nathan, 1996.

Singly, François de, L'Individualisme est un humanisme, La Tour d'Aigues, Éditions de l'Aube, 2005.

Taylor, Charles, Les Sources du Moi. La formation de lidentité moderne, (trad.), Seuil, 1998. 